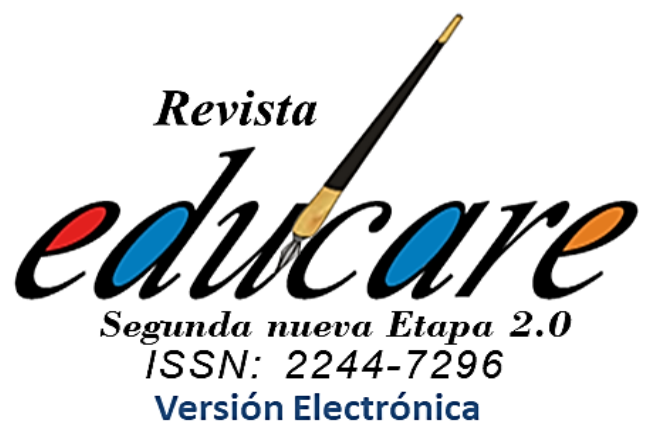

Volumen 24 № 3 Septiembre-Diciembre 2020

(183-204)

Milton Fernando Rosero Duque *

ORCID: https://orcid.org/0000-0002-5702-3524

UNIVERSIDAD CENTRAL DEL ECUADOR ECUADOR

\section{LA IMPORTANCIA DEL MATERIAL, LOS RECURSOS $Y$ ESTÍMULOS APLICADOS COMO JUEGO EN LA ACTIVIDAD FÍSICA}

\author{
THE IMPORTANCE OF THE \\ MATERIAL, RESOURCES AND \\ STIMULI APPLIED AS A GAME IN \\ PHYSICAL ACTIVITY
}

*Magister en Cultura Física y Deportología. Especialista en entrenamiento deportivo, Licenciado en Educación Física Universidad Central del Ecuador, Decano de la Facultad de Cultura Física. Correo electrónico: mfrosero@uce.edu.ec

Recibido:

08-10-2020

Aceptado:

09-11-2020 

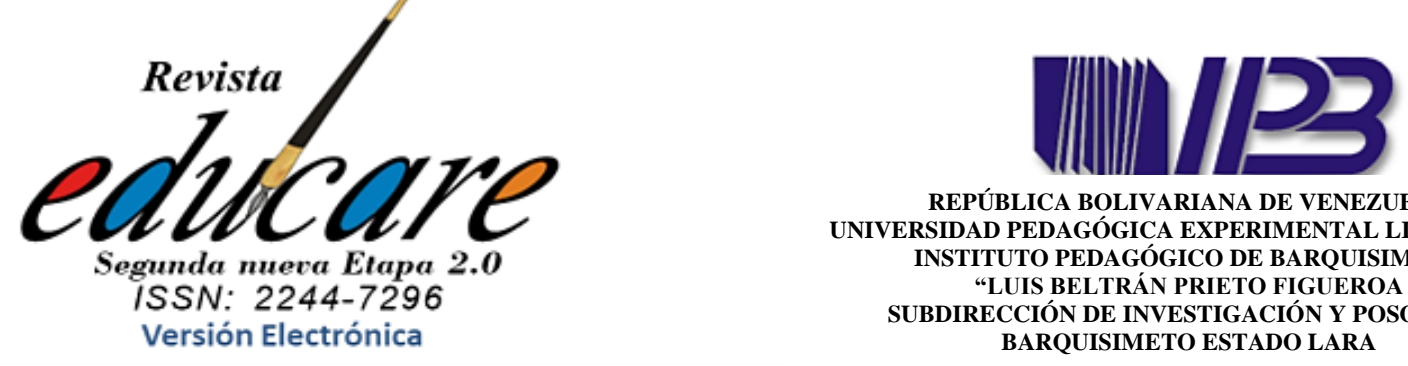

REPÚBLICA BOLIVARIANA DE VENEZUELA UNIVERSIDAD PEDAGÓGICA EXPERIMENTAL LIBERTADOR INSTITUTO PEDAGÓGICO DE BARQUISIMETO "LUIS BELTRÁN PRIETO FIGUEROA

SUBDIRECCIÓN DE INVESTIGACIÓN Y POSGRADO BARQUISIMETO ESTADO LARA

Volumen 24 № 3 Septiembre-Diciembre 2020

(183-204)

\title{
Resumen
}

El presente artículo tiene como propósito dar a conocer los resultados de una investigación realizada con relación a la importancia de material, los recursos y estímulos aplicados como juego en la actividad física. El mismo se abordaron los descriptores materiales y recursos, el Juego y la actividad física, considerando los criterios de Nevado (2008) Jiménez (2006) La UNESCO (2014) entre otros. Este estudio fue realizado siguiendo una perspectiva metodológica cuantitativa, con un nivel descriptivo, en la misma participaron 230 profesionales de Educación Física de diferentes provincias de Ecuador. Se aplicó un instrumento tipo cuestionario con preguntas de alternativas. Los resultados fueron analizado a través de la estadística descriptiva y presentados en gráficos y cuadros. Los resultados permitieron concluir que para profesionales del área de educación física los recursos, los juegos y el estímulo en las actividades físicas son de gran importancia y utilidad.

Palabras clave: Materiales y recursos; actividad física; juegos.

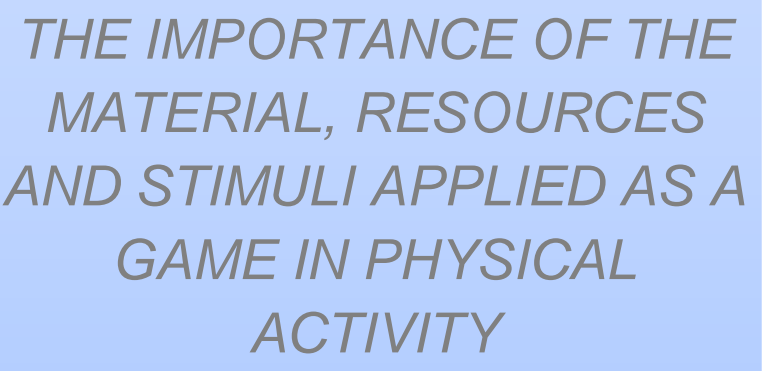

\begin{abstract}
The purpose of this article is to publicize the results of research conducted on the importance of material, resources and stimuli applied as a game in physical activity. It addressed material descriptors and resources, the Game and physical activity, considering the Nevado (2008) Jimenez (2006) UNESCO (2014) criteria among others. This study was carried out following a quantitative methodological perspective, with a descriptive level, involved 230 physical education professionals from different provinces of Ecuador. A questionnaire-type instrument with alternative questions was applied. The results were analyzed through descriptive statistics and presented in charts and charts. The results led to the conclusion that resources, games and encouragement in physical activities are of great importance and usefulness for professionals in the area of physical education.
\end{abstract}

Keywords: Materials and resources; physical activity; games. 


\section{INTRODUCCIÓN}

La importancia del material, los recursos y estímulos aplicados como juego en la Actividad Física son fundamentales ya que permiten realizar experiencias múltiples, mediante los cuales se va alcanzando la comprensión concreta y precisa de los objetivos en las metas propuestas, así como también el proporcionar la capacidad de poder crear, relacionar, y transformar lo que se quiere alcanzar en conocimiento, con un ambiente lleno de emociones, sensaciones y experiencias propias del ser humano mediante actividades lúdicas.

Hay que tomar en cuenta que el material con el que se trabaje debe estar a acorde a las actividades a realizar y muchas veces puede ser lo más sencillo que quizá no lo utilizamos y podemos encontrar en nuestro alrededor, todo esto dependerá del propósito con el cual se va a trabajar analizando: el medio, la edad, las capacidades, el entorno, entre otras. Todo esto permitirá desarrollar habilidades y destrezas en su aspecto físico y cognitivo, lo importante es la adecuada utilización de los materiales que se elija para las actividades.

En cuanto a los estímulos dentro del desarrollo de la actividad física ocupa un lugar fundamental, de ellos van a depender las actividades a realizar. Los estímulos, estarán en función del umbral que la persona tenga y en función de estos, se situara su respuesta adaptativa.

El juego como medio tiene muchas ventajas ya que sobre todo implica alegría, ayuda a desarrollar capacidades físicas en general que aportan nuevas formas de movimiento además, crea posibilidades infinitas de comprender, interpretar e introducirse en el mundo, las personas aprenden a resolver problemas por sí mismos y se socializan apropiadamente, el juego ocupa un lugar esencial y se relaciona con la formación integral.

Con lo anterior, se puede decir que tanto el material como los recursos y los estímulos aplicados como juego en la Actividad Física promueve a que las actividades planteadas en una planificación sean placenteras, ya que el fin que tiene es la diversión, socialización, exploración, creatividad, lo que conlleva a un aprendizaje integral. En este sentido este artículo tiene propósito dar a conocer los resultados de una investigación, la cual tuvo objetivo determinar la importancia de la utilización del material, los recursos y estímulos aplicados en la actividad física como herramientas innovadoras en la estructura de diversas actividades: educativas, empresariales, sociales y culturales, 


\section{FUNDAMENTACIÓN TEÓRICA}

La educación transforma al individuo en un ser social y lo prepara a fin de que pueda trabajar y ser útil a la sociedad; por lo tanto se constituye en la base fundamental para la formación integral que garantice al estudiante la adquisición de habilidades y destrezas, centradas en aprovechar al máximo la aptitudes para la inserción en el contexto social; así lo plantea el Informe de Seguimiento de la Educación Para Todos (EPT) en el mundo elaborado por la UNESCO ( 2014) cuyo propósito es promover cambios en las Políticas Educativas, a partir de la transformación de los paradigmas vigentes, para asegurar aprendizajes de calidad atendiendo al desarrollo humano para todos lo largo de la vida.

Por ello, es pertinente reconocer que, de acuerdo a la postura de la organización internacional anteriormente mencionada, la educación genera cambios, innovaciones y nuevos paradigmas basados en la productividad intelectual teniendo como centro al ser humano. De allí que la educación ecuatoriana es concebida como un proceso de desarrollo del país, debe fomentarse en un esfuerzo armónico, sistemático, coherente de acciones pedagógicas dirigidas a proporcionar a quienes aprenden oportunidades para el logro de objetivos educacionales y puedan encarar con éxito las situaciones presentes y futuras que le disponga la sociedad.

En este sentido, Nevado (2008) menciona que a lo largo de la historia el juego ha sido considerado como un recurso educativo explotado por el hombre desde la antigüedad, y aunque en un principio, no fue tenido en cuenta como parte del proceso educativo, gracias a las diferentes aportaciones de la pedagogía, la psicología, la filosofía, la antropología y la sociología, la educación ha dado un giro aprovechando todas las ventajas que su uso conlleva.

Diferentes estudios han demostrado que el juego incluye pensamiento creativo, solución de problemas, habilidades para aliviar tensiones y ansiedades, capacidad para adquirir nuevos entendimientos, habilidad para usar herramientas y desarrollo del lenguaje y el intelecto permitiendo a niños, jóvenes y adultos hacer juicios solucionar problema desarrollar su creatividad, imaginación e inteligencia

De igual manera, Rodríguez (2006) expone que el juego es una actividad humana y vivencial que promueve la evolución íntegra de quienes se involucran en él, claro que cuando se habla de juego no todos piensan lo mismo, se cree que es pura distracción y pérdida de tiempo; pero además resulta ser una actividad que desarrolla actitudes, habilidades y 
capacidades de beneficio para la educación y de esto último surge la importancia que poseen los juegos como estrategias didácticas dentro del campo educativo.

En torno a esto Jiménez (2006) conceptualiza al juego como una técnica participativa de la enseñanza encaminada a desarrollar en los estudiantes; métodos de dirección y conducta correcta, que estimula la disciplina con un adecuado nivel de decisión y autodeterminación; es decir, no sólo propicia la adquisición de conocimientos y el desarrollo de habilidades, sino que además contribuye al logro de la motivación por las asignaturas; y constituye una forma de trabajo docente que brinda una gran variedad de procedimientos para el entrenamiento de los estudiantes en la toma de decisiones para la solución de diversas problemáticas.

De igual manera para Piaget (citado por Nevado, 2008), el juego cumple un rol esencial en la formación de la personalidad y es de gran importancia para el desarrollo de la inteligencia, sirve también como equilibrio de la afectividad y permite al niño su socialización y su incorporación a su entorno. De esta manera, el juego se constituye en una herramienta que le brinde al niño la posibilidad a la práctica educativa como elemento indispensable en el aprendizaje y en su desarrollo integral. De la misma forma, el juego tiene sentido recreativo como pedagógico, porque la jocosidad trae consigo la diversión y con ella los aprendizajes.

El juego como actividad espontánea y voluntaria que proporciona un cierto grado de alegría es un sistema que desarrolla en quien lo practica la capacidad de crear con libertad, a pesar de los lineamientos que se puedan generar para practicarlo o desarrollarlo. Además, puede estar presente en todo tipo de actividades, en todos los procesos de aprendizaje, en los diferentes campos del conocimiento y, como se ha mencionado, en la construcción de la vida misma (Melo, y Hernández, 2014). El juego es el medio ideal para el trabajo principalmente en grupos.

Dentro del currículo de Educación Física ecuatoriano, las actividades lúdicas entre ellas el juego, forman parte del primer bloque curricular de contenidos. De acuerdo el Ministerio de Educación (2017) el juego está enmarcado dentro de las prácticas corporales y son concebidos como producciones culturales, con estructuras reconocibles en sus contextos de creación.

Los juegos presentan estructuras, lógicas y objetivos, que en la enseñanza de la Educación Física deben explicitarse para garantizar su comprensión y apropiación. Eso permite que niños, niñas y jóvenes puedan jugar o participar de los juegos, establecer vínculos con otros y con la cultura del movimiento y, además, generar procesos creativos. 


\section{Material y Recursos}

En la triada didáctica los materiales y los recursos juegan un papel muy importante, ellos representan el soporte y la herramienta del docente para enseñar y del estudiante para aprender. Estas herramientas entran dentro de la definición de las estrategias como el componente esencial del proceso de aprendizaje y enseñanza. Son el sistema de actividades (acciones y operaciones) que permiten la realización de una tarea con la calidad requerida dada la flexibilidad y adaptabilidad que ellas ofrecen en su empleo a las condiciones existentes (Serrano, 2014).

De allí que los materiales y recursos junto con todas las estrategias didácticas representan un sistema de acciones y operaciones, tanto físicas como mentales, que facilitan la confrontación (interactividad) del sujeto que aprende con el objeto de conocimiento, y la relación de ayuda y cooperación con otros colegas durante el proceso de aprendizaje (interacción) para realizar una tarea con la calidad requerida. Estas estrategias constituyen herramientas de mediación entre el sujeto que aprende y el contenido de enseñanza que el docente emplea conscientemente para lograr determinados aprendizajes (Serrano, 2014).

Para Molina, Martínez y Gómez (2017) los materiales y recursos se convierten en transmisores de una serie de experiencias y conocimientos. En tanto para Vargas (2017) los recursos y materiales deben considerar el grupo al que va dirigido, con la finalidad que ese recurso realmente sea de utilidad. Entre las funciones que tienen los recursos didácticos se encuentran: a) proporcionar información, b) cumplir un objetivo, c) guiar el proceso de enseñanza y aprendizaje, d) contextualizar a los estudiantes, e) factibilidad la comunicación entre docentes y estudiantes, f) acercar las ideas a los sentidos, g) motivar a los estudiantes.

Para Muñoz Díaz (2003), los recursos y materiales didácticos para la educación física son aquellos elementos materiales y a todas las estrategias que pueden ser utilizados por los docentes que le sirvan como soporte o complemento en su tarea para llevarla a la práctica, mejorarla y reconducirla eficazmente.

Por su parte Trujillo (2010), menciona que por materiales se definen aquellos han sido elaborados con una finalidad explícita, mientras que los recursos didácticos toman su verdadero valor pedagógico en el momento en el que los docentes deciden, desde la metodología, integrarlos. Los recursos y materiales utilizados cumplen distintas finalidades, siendo las más relevantes las siguientes:

- Son un medio entre la teoría impartida y la práctica deportiva. 
- Motivan a los participantes a la hora de realizar las tareas.

- Favorecer la comunicación y comprensión de participantes.

- Son un elemento auxiliar eficaz.

- Posibilita la auto instrucción.

Los materiales didácticos y/o recursos deben en forma general una utilidad pedagógica, es decir, una función tanto especifica cómo general y cumplir en el mayor de los casos con el principio de polivalencia. Para Rodríguez (2005) en Moreno (2017) refiere que los materiales que se utilicen en la educación deben cumplir tres funciones primordiales:

1. Función de apoyo al aprendizaje: los materiales favorecen la interiorización de los contenidos de una manera eficaz y significativa dentro del proceso de adquisición de aprendizaje. Los materiales juegan un papel esencial al ser los canalizadores en la selección y almacenamiento de la información.

2. Función estructuradora: ayudan a la hora de ordenar y estructurar toda la información que reciben, de manera que lo abstracto se verá transformado en conocimientos más concretos y accesibles, consiguiendo con ello, que los alumnos obtengan unos aprendizajes más específicos y concretos.

3. Función motivadora: todo recurso material tiene que ser llamativo, fuente de interacción y favorecedora del aprendizaje de forma agradable, divertida y placentera, donde el niño no conciba en sí mismo el objetivo principal que persigue, que es el aprendizaje, sino que considere toda actividad educativa como una ampliación más de su vida diaria.

Con base en lo señalado, Bautista (2010) establece una serie de características que deben tener los materiales en relación a la función complementaria que realiza en la actividad educativa, como:

- Carácter motivador; ya que, según la forma, textura, color y características particulares del material, despertara el interés y curiosidad para su utilización.

- Carácter polivalente; los materiales pueden ser utilizados como elementos en varias actividades escolares y como elementos de diferentes juegos.

- Carácter colectividad; su utilización puede ser individual o colectiva.

- Carácter de accesibilidad; estar organizado y disponible para la libre elección de los alumnos. 


\section{Actividad Física}

La OMS (2020) define la actividad física como cualquier movimiento corporal producido por los músculos esqueléticos, con el consiguiente consumo de energía. Ello incluye las actividades realizadas al trabajar, jugar y viajar, las tareas domésticas, las actividades educativas y recreativas.

La expresión «actividad física» no se debería confundir con «ejercicio», que es una subcategoría de actividad física que se planea, está estructurada, es repetitiva y tiene como objetivo mejorar o mantener uno o más componentes del estado físico. La actividad física tanto moderada como intensa es beneficiosa para la salud.

Además del ejercicio, cualquier otra actividad física realizada en el tiempo de ocio, para desplazarse de un lugar a otro o como parte del trabajo, también es beneficiosa para la salud. La actividad física tanto moderada como intensa es beneficiosa para la salud.

En palabras de Annicchiarico (2002) la actividad física hace referencia al movimiento, la interacción, el cuerpo y la práctica humana. Tiene tres dimensiones: biológica, personal y sociocultural. Desde la dimensión biológica (la más extendida) se define como cualquier movimiento corporal realizado con los músculos esqueléticos que lleva asociado un gasto de energía.

En este mismo sentido para Bouchard et al. (1996) en Vidarte Claros et al. (2011) considera que actividad física se retoma desde la promoción de la salud a través de la intervención de profesionales en esta área, instituciones sanitarias y educativas como un medio intercesor fundamental que contribuye en la obtención de resultados significativos en pro de la evolución y mejoramiento de la calidad de vida.

En relación con lo señalado, Annicchiarico (2002) hace referencia que la actividad física, es aquella que integra las tres dimensiones citadas: "La actividad física es cualquier movimiento corporal intencional, realizado con los músculos esqueléticos, que resulta en un gasto de energía y en una experiencia personal, y nos permite interactuar con los seres y el ambiente que nos rodea" (s/p).

La mayor repercusión de la actividad física está asociada a la salud la cual de acuerdo Annicchiarico (2002) debe estar orientada en lo siguiente:

- Ser moderada (permitir llevar una práctica constante durante largo tiempo).

- Vigorosa (intensidad que produzca sudoración y jadeo en la respiración). 
- Habitual y frecuente, de manera que forme parte del estilo de vida.

- Orientada al proceso de práctica, más que a un resultado o alto rendimiento.

- Satisfactoria.

- Social; que permita relaciones entre las demás personas.

De igual manera si está orientada a la diversión como aspecto lúdico, debe ser acorde con la edad y características psicofísicas de los practicantes, y las actividades deben ser variadas y adaptadas a las características personales. En esta misma perspectiva Rincic y Valero (2002) exponen en cuatro aspectos básicos los beneficios de la actividad física:

\section{Figura 1}

Beneficios de la actividad física

\begin{tabular}{|c|c|c|c|c|c|c|}
\hline \begin{tabular}{|l|} 
Aparato \\
muscular \\
esquelético
\end{tabular} & $\begin{array}{l}\text { Postura } \\
\text { correcta } \\
\text { mayor }\end{array}$ & $\begin{array}{l}\text { Masas } \\
\text { musculares } \\
\text { simétricamente } \\
\text { tónica }\end{array}$ & $\begin{array}{l}\text { Movilidad } \\
\text { articular }\end{array}$ & & & \\
\hline $\begin{array}{l}\text { Aparato } \\
\text { cardiovascular }\end{array}$ & $\begin{array}{l}\text { Disminución } \\
\text { de la } \\
\text { frecuencia } \\
\text { cardíaca }\end{array}$ & $\begin{array}{l}\text { Rápida } \\
\text { recuperación } \\
\text { después del } \\
\text { esfuerzo }\end{array}$ & $\begin{array}{l}\text { Óptima } \\
\text { contracción } \\
\text { sistólica }\end{array}$ & $\begin{array}{l}\text { Presión } \\
\text { arterial } \\
\text { favorable }\end{array}$ & $\begin{array}{l}\text { Disminución } \\
\text { de la } \\
\text { frecuencia } \\
\text { respiratoria }\end{array}$ & $\begin{array}{l}\text { Incremento } \\
\text { del } \\
\text { volumen } \\
\text { pulmonar }\end{array}$ \\
\hline $\begin{array}{l}\text { Sistema } \\
\text { endocrino y } \\
\text { metabólico }\end{array}$ & $\begin{array}{l}\text { Relación } \\
\text { peso talla } \\
\text { favorable }\end{array}$ & $\begin{array}{l}\text { Aumento de la } \\
\text { masa magra } \\
\text { activa }\end{array}$ & $\begin{array}{l}\text { Reducción } \\
\text { de la masa } \\
\text { grasa }\end{array}$ & $\begin{array}{l}\text { Regulación } \\
\text { del control } \\
\text { del apetito }\end{array}$ & $\begin{array}{l}\text { Correcta } \\
\text { asimilación } \\
\text { de gases }\end{array}$ & \\
\hline $\begin{array}{l}\text { Personalidad y } \\
\text { comportamient }\end{array}$ & $\begin{array}{l}\text { Buen } \\
\text { control } \\
\text { emotivo }\end{array}$ & $\begin{array}{l}\text { Buena } \\
\text { adaptabilidad }\end{array}$ & $\begin{array}{l}\text { Buena } \\
\text { capacidad de } \\
\text { socialización }\end{array}$ & $\begin{array}{l}\text { Mejor } \\
\text { tolerancia } \\
\text { a la } \\
\text { frustración }\end{array}$ & $\begin{array}{l}\text { Válida } \\
\text { autoestima }\end{array}$ & \\
\hline
\end{tabular}

Nota: Tomado de Rincic y Valero (2002).

\section{Materiales y recursos para la actividad física}

De acuerdo con lo anterior expuesto y haciendo referencia a Frutos (2014) El concepto de material o recurso didáctico en la actividad física tiene un carácter instrumental. Se trata de cualquier dispositivo o equipo que se utiliza para transmitir información; entre los materiales se pueden nombrar pelotas, cuerdas, colchonetas, palas, raquetas entre otros.

Estos son materiales específicos y que por lo general son utilizados en las clases de educación física. De acuerdo a Blández (1998) en Frutos (2014) pueden ser divididos en cuatro categorías de gran connotación lúdico y deportivo: 
a) Los de gimnasio: tienen origen en los movimientos gimnásticos de los siglos XIX y $\mathrm{XX}$, uno de sus principios es imitar la gimnasia natural, por ejemplo, las colchonetas pueden tener cierta similitud con las praderas; las espalderas con los grandes árboles por los que se podría trepar; o el potro con las piedras que nos servían para saltar de un lado a otro. Otros materiales que se pueden utilizar en esta categoría son la escalera, las cuerdas colgadas del techo, el plintom, el trampolín, la barra de equilibrio y el banco sueco.

b) De psicomotricidad: los materiales y recursos que se emplean en esta categoría se deben elegir en función de los contenidos a desarrollar, por ejemplo: para actividades de coordinación se pueden utilizar pelotas de diversos tamaños, texturas y colores, gomas elásticas, cuerdas, aros, bolos, raquetas, pompones o grandes materiales como bloques de espuma, ladrillos de plásticos o túneles de gateo. Para actividades encaminadas al desarrollo del esquema corporal se pueden utilizar figuras de colores, huellas de manos y pies, espejos, o muñecos articulados. Para actividades de expresión corporal y ritmo son útiles los panderos, claves, panderetas, maracas, castañuelas, triángulos.

c) Los de deportes: entre los que tenemos que diferenciar los siguientes: individuales: vallas, testigos, pesas, (atletismo); colchonetas, aros, cuerdas, (gimnasia) equipo: stick y bolas de hockey (hockey); raquetas y volantes (badminton); balones de iniciación y oficiales (fútbol, baloncesto, voleibol, balonmano) A estos materiales citados habría que añadir distintos accesorios como son las porterías, canastas o las redes que posibilitan el deporte en su totalidad. Otros: indiacas, frisbees y patines.

\section{El Juego como actividad física}

El juego representa la forma natural y espontanea del ser humano para expresarse y socializar desde los primeros años de vida. Es por ello que realizar actividades físicas con base en el juego le da la oportunidad a niños, adolescentes y adultos darle movilidad a su cuerpo y cuidar la salud mientras se divierte, desde un contexto espontaneo y lúdico; como dice Zabalza, (2006):

El juego es una actividad espontánea, voluntaria y libremente elegida, este no debe admite exigencias externas, el participante debe sentirse libre de actuar como quiera, si el juego tiene imposiciones deja de ser espontáneo, libre; además el participante pierde interés en él por ello debe dejar que fluya. Estas características de la propia dinámica del juego son las que se 
utilizan en muchas ocasiones para la creación de determinados hábitos sociales que permiten a las personas vivir en comunidad, en donde reglas, normas, libertad, autonomía y responsabilidad se conjugan como fórmulas para la creación de espacios de convivencia y de paz (p.120).

Desde la acción natural del juego, el ser humano se activa corporalmente de manera espontánea. Es una forma de actividad física que le va a permitir tanto al niño como al adulto como lo refiere Farre (2016) la expresión de su energía, la necesidad de movimiento, de comunicación, de resolución de problemas; ayuda a desarrollar la creatividad, a poner en práctica la imaginación y, con todo esto, poder adquirir formas complejas que van a propiciar el desarrollo de competencias. En el juego, van a variar no solo la complejidad y el sentido, sino también la forma de participación en la que se llevan a cabo, que van desde la individual, donde los procesos de atención y concentración son básicos, los juegos en pareja, donde se ponen en práctica habilidades de negociación y mediación y, los juegos colectivos donde es necesario llegar a acuerdos y poner en práctica habilidades de colaboración.

En este mismo sentir, la UNESCO (1980) señala que efectivamente, el juego es vital; condiciona un desarrollo armonioso del cuerpo, de la inteligencia y de la afectividad,

El juego constituye por lo demás una de las actividades educativas esenciales y merece entrar por derecho propio en el marco de la institución escolar, mucho más allá de los jardines de infantes o escuelas de párvulos donde con demasiada frecuencia queda confinado. (UNESCO 1980, p 35)

En virtud de lo referido, Rodríguez y Monroy (2015) haciendo alusión a Huizinga (1990) definen a los juegos con fines de actividad física:

Como acciones libres ejecutada como sí y sentida como situada fuera de la vida corriente, pero que, a pesar de todo, puede absorber por completo al jugador, sin que haya en ella ningún interés material ni se obtenga en ella provecho alguno, que se ejecuta dentro de un determinado tiempo y de un determinado espacio, que se desarrolla en un orden sometido a reglas y que da origen a asociaciones que propenden a rodearse de misterio o a disfrazarse para destacarse del mundo habitual. (p 52)

Tomado en cuenta la definición y el desarrollo de las mismas Rodríguez y Monroy (2015) establecer una serie de características del juego:

- Es una actividad libre (el juego por mandato no es propiamente un juego) 
- Carácter desinteresado, en relación con la función cultural del mismo

- limitación temporal y espacial.

- Presencia de un cierto grado de incertidumbre o azar, lo cual genera tensión en el desarrollo del mismo e implica la existencia de una serie de reglas

- Tendencia a perdurar el equipo de jugadores (en el caso de juegos por equipos) aun después de terminado.

- Frecuente misterio y secretismo en el desarrollo del mismo.

Desde esta misma perfectiva Roncancio y Sichacá (2009) hacen reconocimiento del juego como actividad física, es una práctica que contribuye a proteger el bienestar físico, mental y social, que trasciende más allá de lo biológico y debe ser entendida para satisfacer necesidades inherentes al ser humano en el reencuentro espiritual, en el reconocimiento con la corporeidad y en el disfrute del "placer" que genera el cuerpo en movimiento, así como una práctica motivadora y socializadora de los sujetos.

El juego como actividad física señala Zampa (2007) ocupa un lugar muy importante en el campo pedagógico, psicológico y artístico no sólo para los niños sino también para los adultos. En tal sentido el objetivo del juego no puede ser otra cosa que jugar el permite que la persona interactúe, asimile, comprenda espontánea y orgánicamente las posibilidades y los límites que le ofrece determinado material o propuesta o en definitiva la realidad. Por lo tanto, el juego es una necesidad vital que contribuye al equilibrio humano, es una actividad exploradora, de aventura y experiencia, es medio de comunicación y de liberación, es un proceso de educación completa, indispensable para el desarrollo físico, intelectual y social de las personas.

\section{El estímulo aplicado a la actividad física}

Dentro del desarrollo de la condición física de jóvenes y adultos, los estímulos ocupan un lugar fundamental (Giménez y Díaz, 2002), de ellos van a depender las actividades y cargas que determinan el entrenamiento. Los estímulos, estarán en función del umbral que la persona tenga y en función de estos, se situara su respuesta adaptativa. En virtud de ello el Diccionario de las Ciencias del Deporte (1992) en Giménez y Díaz, (2002) conceptualiza "el estímulo se refiere a un acontecimiento, interno o externo al organismo, que actúa sobre estados de este y que puede producir cambios en él” (p.97). Rosa y Del Río (1999), citando en Giménez y Díaz 
(2002) lo definen como aquel "agente externo que provoca una acción determinada como respuesta" (p.158).

Para Giménez y Díaz, (2002), el estímulo en la actividad física es sinónimo de ejercicio físico, por lo que su conocimiento y adecuada utilización es básico para el profesor-entrenador. Ahora en el campo educativo, la actividad física está determinada por los estímulos y su intensidad, siendo de vital importancia la pausa, que debe estar en consonancia con los ejercicios.

De forma general los autores citados presentan tres tipos de estímulos según su intensidad:

- Estímulos de intensidad máxima: Se caracterizan por una frecuencia cardiaca alta (180 p/m) y deuda de oxígeno del 80\% ó 95\%. Tipos de esfuerzos: saltos, lanzamientos.

- Estímulos de intensidad submáxima: Se caracteriza por una frecuencia cardiaca situada entre 140 y 150 p/m. y una deuda de oxígeno del 50\% al 80\%. Tipos de esfuerzos: medio fondo, deportes de equipo.

- Estímulos de intensidad media: El más adecuado para la primaria y con unas características de 140 p/m. y deuda de oxígeno muy baja de un 7\% y 10\%, esfuerzos de larga duración y baja intensidad.

En virtud de lo referido se hace importante considerar el estímulo dentro de las prácticas de la actividad física, por todo el beneficio que puede generar además que incrementa la disposición y el interés de los sujetos por ser parte de esta no solo dentro del contexto académico sino como agente que incrementa la salud física y mental.

\section{METODOLOGÍA UTILIZADA}

El estudio realizado se enmarcó dentro del paradigma positivista a través de una investigación cuantitativa, al respecto Hernández, Fernández y Baptista (2010), la define como aquella en la que "el investigador utiliza su diseño para analizar la certeza de la hipótesis formulada en un contexto en particular o para aportar evidencia respecto a los lineamientos de la investigación (p.184).

El Diseño de la Investigación fue de campo de carácter descriptivo. Tamayo y Tamayo (2005.) señalan que en la investigación descriptiva "se resalta la aplicabilidad de procedimientos dirigidos a desglosar y desintegrar los hechos en partes para conocer el estado 
en que se encuentran. Este proceso permite reconocer las características o cualidades del objeto y situación tratada" (p.53).

En cuanto la variable en la investigación esta representa un concepto de vital importancia, está conforma por enunciados de un tipo particular. Las variables se refieren a las propiedades de la realidad que varían "cuya variación es susceptible de medirse" (Hernández et al., p.43). En este sentido la variable del estudio es "la importancia del material, los recursos y estímulos aplicados como juego en la actividad física".

La muestra estuvo conformada por 203 profesionales de Educación Física 137 hombres y 66 mujeres con preparación académica de Tercer Nivel (153) Cuarto Nivel (44) y Doctorado (4) distribuidos de acuerdo a su puesto laboral de siguiente manera Inicial (2) Preparatoria (6) Educación General Básica (97) Bachillerato General Unificado (59) y Superior (39). A los mismos se les aplico un cuestionario contentivo de 12 preguntas tipo escala de Liker

Las provincias que participaron es esta investigación fueron:

\section{Tabla 1}

Provincias que participaron en la investigación

\begin{tabular}{lllll}
\hline Pichincha & Carchi & Manabí & Quito & Pastaza \\
Guayas & Esmeraldas & Azuay & Murillo & Santa Cruz \\
Tungurahua & Riobamba & El Oro & Galápagos & Tungurahua \\
Esmeraldas & Chimborazo & Trujillo & Imbabura & \\
Cotopaxi & México & Santo Domingo de los & Nueva Unión & Bolivar \\
& & Sáchilas & \\
\hline
\end{tabular}

Nota: Elaboración propia.

\section{RESULTADOS}

A continuación, se presentan los resultados de la encuentra, los mismos fueron procesados siguiendo la estadística descriptiva y presentado en tablas y gráficos con frecuencia y porcentual, siguiendo los objetivos propuestos en la investigación.

Con relación a la importancia la importancia del material, los recursos y estímulos aplicados como juego en la actividad física se determinó lo siguiente: 


\section{Tabla 2}

Importancia del uso de los materiales y recursos en la actividad física

\begin{tabular}{lcc}
\hline \multicolumn{1}{c}{ Categoría } & Frecuencia & Porcentaje \\
\hline No es importante & 0 & 0 \\
Poco importante & 3 & 1,5 \\
Importante & 61 & 30 \\
Muy importante & 139 & 68,5 \\
TOTAL & $\mathbf{2 0 3}$ & $\mathbf{1 0 0}$ \\
\hline
\end{tabular}

Nota: Elaboración propia.

\section{Gráfico 1}

Distribución de la importancia del uso de los materiales y recursos en la actividad física

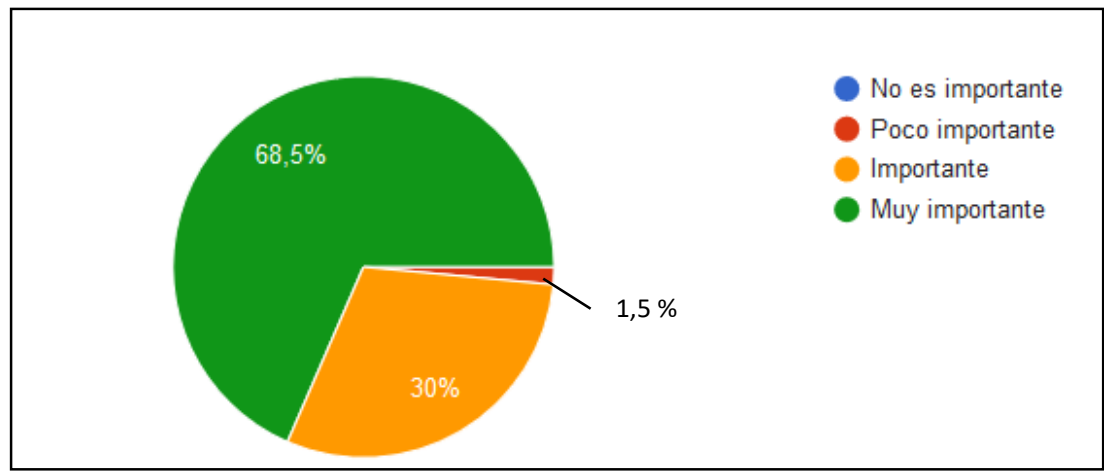

Se observa en la tabla 2 y gráfico 1 que, del total de la muestra, el 139 que representa el 68,5\% consideran que es muy importante el uso de materiales en la Actividad Física, 61 de ellos que representa el 30\% mencionan que es importante y 3 que representa al 1,5\% responden que es poco importante. Por tal razón y de acuerdo a los porcentajes obtenidos de forma significativa se puede determinar que es muy importante el uso de materiales en la Actividad Física.

Al preguntarle a los encuestados sobre importancia tiene para ellos el uso de los estímulos en la Actividad Física, se aprecia en el cuadro y grafico lo siguiente: 


\section{Tabla 3}

Importancia del uso de estímulos en la Actividad Física

\begin{tabular}{lcc}
\hline \multicolumn{1}{c}{ Categoría } & Frecuencia & Porcentaje \\
\hline No es importante & 0 & 0 \\
Poco importante & 3 & 1,5 \\
Importante & 43 & 21,2 \\
Muy importante & 157 & 77,3 \\
TOTAL & $\mathbf{2 0 3}$ & $\mathbf{1 0 0}$
\end{tabular}

Nota: Elaboración propia.

\section{Gráfico 2}

Distribución de la importancia del uso de los estímulos en la educación física

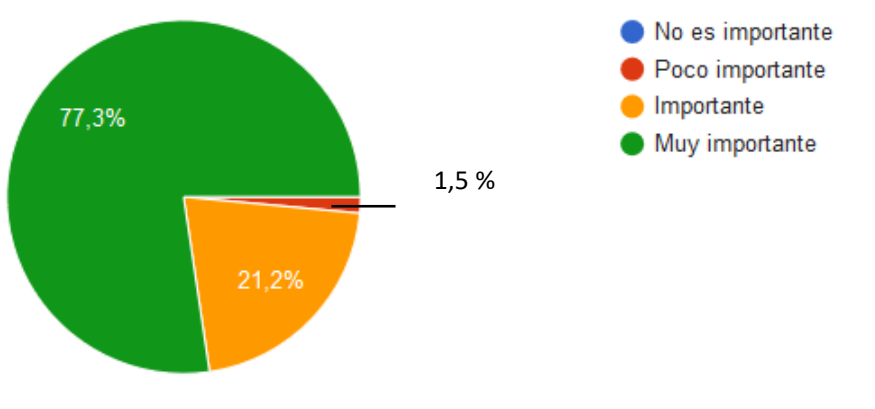

Se puede observar en la tabla 3 y gráfico 2 que el 157 de la muestra el cual representa el 77,3\% consideran que es muy importante la importancia del uso de estímulos en la Actividad Física, 43 que representa el 21,2\% mencionan que es importante y 3 que representa al 1,5\% responden que es poco importante. Por tal razón y de acuerdo a los porcentajes obtenidos de forma significativa se puede determinar que es muy importante el uso de estímulos en la Actividad Física 


\section{Tabla 4}

Importancia del juego en la Actividad Física

\begin{tabular}{lcc}
\hline \multicolumn{1}{c}{ Categoría } & Frecuencia & Porcentaje \\
\hline No es importante & 1 & 0,5 \\
Poco importante & 2 & 1 \\
Importante & 27 & 13,3 \\
Muy importante & 173 & 85,2 \\
TOTAL & $\mathbf{2 0 3}$ & $\mathbf{1 0 0}$
\end{tabular}

Nota: Elaboración propia.

\section{Gráfico 3}

Distribución de la Importancia del juego en la Actividad Física

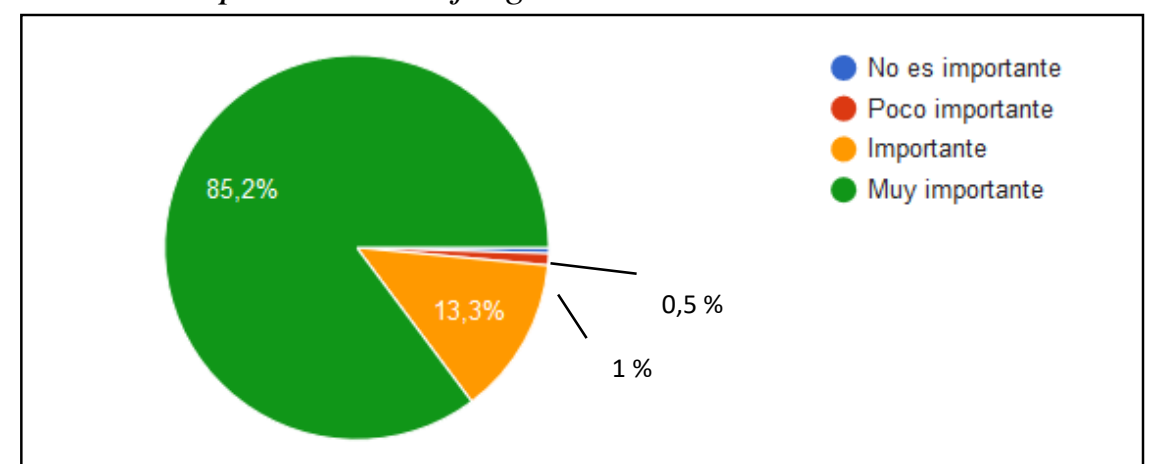

Se puede observar en la tabla 4 y gráfico 3 que del total de la muestra encuestada el 173 que representa el $85,2 \%$ consideran que es muy importante la importancia del juego en la Actividad Física, 27 que representa el 13,3\% mencionan que es importante, 2 que representa al $1 \%$ responden que es poco importante y 1 que representa el $0,5 \%$ contesta q no es importante. Por tal razón y de acuerdo a los porcentajes obtenidos de forma significativa se puede determinar que es muy importante el juego en la Actividad Física.

\section{Tabla 5}

Importancia de la utilización de los materiales aplicados como juego en la Actividad Física

\begin{tabular}{lcc}
\hline \multicolumn{1}{c}{ Categoría } & Frecuencia & Porcentaje \\
\hline No es importante & 0 & 0 \\
Poco importante & 3 & 1,5 \\
Importante & 68 & 33,5 \\
Muy importante & 132 & 65 \\
TOTAL & $\mathbf{2 0 3}$ & $\mathbf{1 0 0}$ \\
\hline
\end{tabular}

Nota: Elaboración propia. 


\section{Gráfico 4}

Distribución de la Importancia de la utilización de los materiales aplicados como juego en la Actividad Física

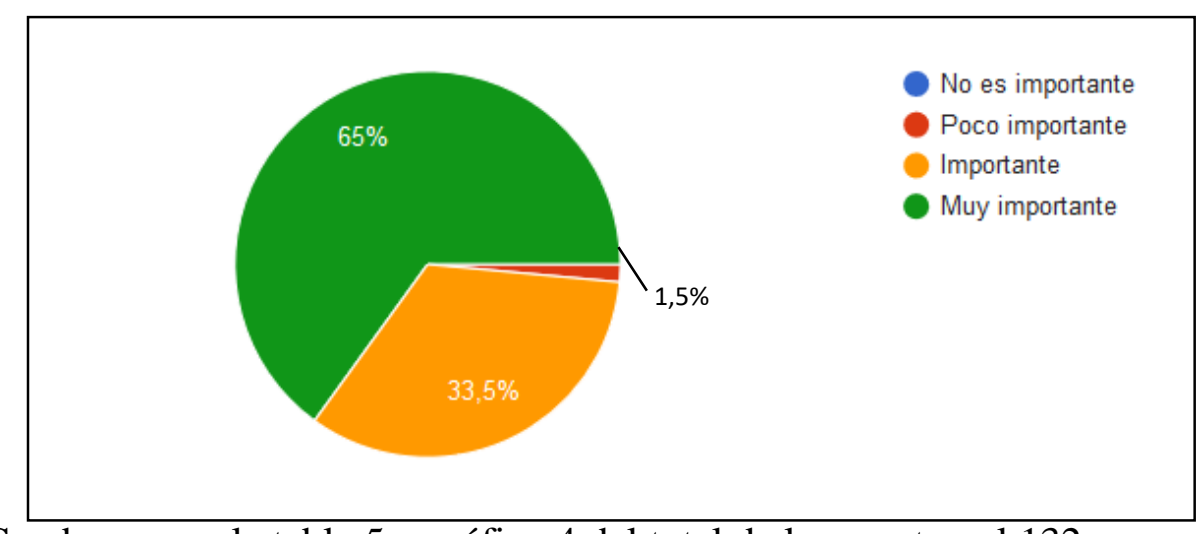

Se observa en la tabla 5 y gráfico 4 del total de la muestra, el 132 que representa el 65\% consideran que es muy importante importancia de la utilización de los materiales aplicados como juego en la Actividad Física, 68 que representa el 33,5\% mencionan que es importante y 3 que representa al 1,5\% responden que es poco importante. Por tal razón y de acuerdo a los porcentajes obtenidos de forma significativa se puede determinar que es muy importante la utilización de los materiales aplicados como juego en la Actividad Física.

\section{Tabla 6}

Recursos aplicados como juego en la Actividad Física

\begin{tabular}{lcc}
\hline \multicolumn{1}{c}{ Categoría } & Frecuencia & Porcentaje \\
\hline Nunca & 0 & 0 \\
A veces & 20 & 9,9 \\
Frecuentemente & 67 & 33 \\
Siempre & 116 & 57,1 \\
TOTAL & $\mathbf{2 0 3}$ & $\mathbf{1 0 0}$
\end{tabular}

Nota: Elaboración propia. 


\section{Gráfico 5}

Distribución de Recursos aplicados como juego en la Actividad Física

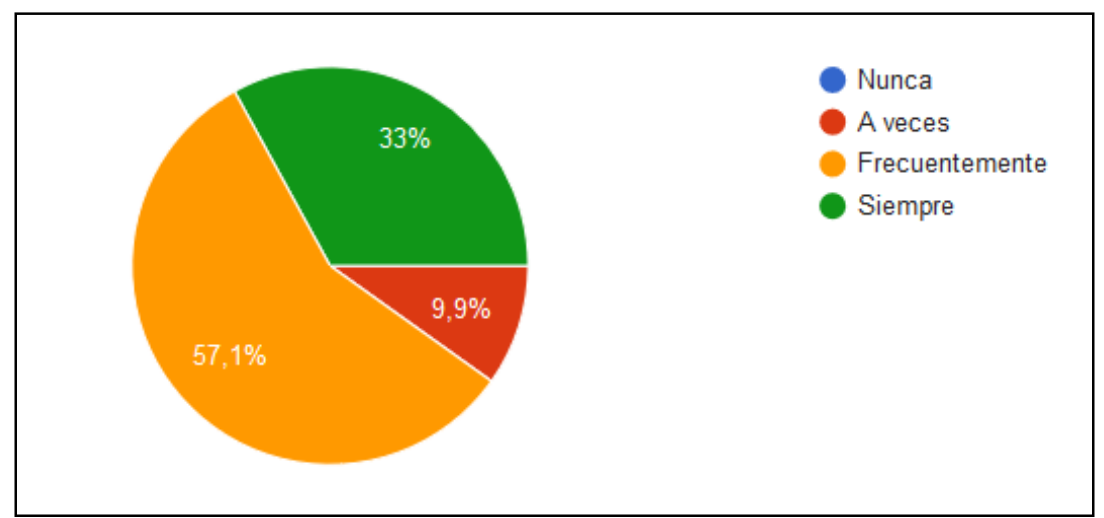

Se aprecia en la tabla 6 y gráfico 5 que, del total de la población, 116 que representa el 57,1\% consideran que siempre utilizan recursos aplicados como juego en la Actividad Física, 67 que representa el 33\% mencionan frecuentemente y 20 que representa al 9,9\% responden a veces. Por tal razón y de acuerdo a los porcentajes obtenidos de forma significativa se puede determinar que siempre utilizan recursos aplicados como juego en la Actividad Física.

\section{CONSIDERACIONES FINALES}

El resultado determina de una manera concluyente la importancia en primer lugar que tiene para los profesionales de Educación Física los materiales y recursos en la actividad física, esto permite determinar que para ellos las actividades por sí sola no es suficiente, por lo tanto, se hace necesario algún tipo de material o recurso, todos aquellos que favorezca la labor docente en el desarrollo de su práctica profesional en las actividades física.

De igual manera impone la importancia que tiene para los profesionales encuestados del uso de estímulos en la actividad física, dado que de acuerdo a la actitud y la aptitud de los participantes el entrenador puede intensificar la actividad y por lo tanto generar mayor beneficio.

Por otro lado, se concluye de manera explícita la importancia que tiene el juego en la actividad física dado que este ocupa un lugar muy importante en el campo pedagógico, psicológico y artístico no sólo para los niños sino también para los adultos, el juego representa la actividad natural del ser humano no solo para ejercitarse sino también por el grado de recreación, bienestar y salud que este genera. También los resultados brindan una singular 
importancia al uso tanto de recursos y materiales dentro de las estrategias lúdicas deportivas en la actividad física, De allí que los materiales y recursos junto con todas las estrategias didácticas representan un sistema de acciones y operaciones, tanto físicas como mentales, que facilitan la interactividad entre el estudiante con el objeto de conocimiento, y la relación de ayuda y cooperación con otros durante el proceso de aprendizaje para realizar las accione y cumplir con ellas con la calidad requerida.

\section{REFERENCIAS}

Annicchiarico, R. (2002) La actividad física y su influencia en una vida saludable. https://www.efdeportes.com/efd51/salud.htm

Bautista, J. (2010). Los materiales como mediadores. www.investigalog.com/el_juego_como_metodo_didactico/tema8losmaterialescomomed iadores/

Farre, C. (2016) Educación XXI. Aprender Jugando. https://mx.unoi.com/2015/06/01/aprenderjugando/

Frutos, J. (2014) Recursos y materiales didácticos específicos del área de educación física. https://www.preparadores.eu/temamuestra/Maestros/EF.pdf

Gimenez, F. y Diaz, M. (2002). Diccionario de Educación Física en Primaria (II). https://www.efdeportes.com/efd51/dicc.htm\#: :text=Rosa\%20y\%20Del\%20R\%C3\%A Do\%20(1999,b\%C3\%A1sico\%20para\%20el\%20profesor\%2Dentrenador

Glosario de términos especializados. (2018). Glosario Educación Física. Estímulo. Recuperado de. https://glosarios.servidor-alicante.com/educacion-fisica/estimulo

Hernández, R., Fernández, C y Baptista, P. (2010). Metodología de la Investigación. Cuarta edición. Editorial Mc GRaw Hill. México.

Jiménez, E. (2006). La capacidad creadora. Editorial Graó, vol. III, Barcelona

Molina, P., Martinez, A. y Gómez F. (2017) El uso de materiales curriculares en educación física: racionalidad y desarrollo profesional del profesorado. Revista de Educación Física de la Universidad de Antioquia, 6(3), pp. 9-28. https://revistas.udea.edu.co/index.php/educacionfisicaydeporte

Melo. M. y Hernández, R. (2014). El juego y sus posibilidades en la enseñanza de las ciencias naturales. Innovación educativa (México, DF), 14(66), 41-63. Recuperado en 15 de 
junio de 2020, de http://www.scielo.org.mx/scielo.php?script=sci_arttext\&pid=S1665$26732014000300004 \& \operatorname{lng}=$ es\&tlng=es.

Ministerio de Educación (2017) Currículo Básico Nacional del Ecuador. https://educacion.gob.ec/curriculo/

Moreno, L. (2017). La Influencia de los materiales manipulativos durante el proceso de enseñanza/aprendizaje en segundo ciclo de educación infantil. Tesis Doctoral Universidad de Murcia Facultad De Educación. https://www.tdx.cat/bitstream/handle/10803/405577/TFJML.pdf?sequence=1\&isAllowe $\underline{\mathrm{d}=\mathrm{y}}$

Mundial, O. (2020). Organización Mundial de la Salud. Actividad Física. https://www.who.int/es/news-room/fact-sheets/detail/physical-activity

Muñoz Díaz, J. (2003). Posibilidades de aplicación didáctica de los materiales y recursos de EF en Educación Primaria. Lecturas: EF y Deportes, 65. https://www.efdeportes.com/efd65/material.htm

Nevado, CH. (2008). El componente lúdico en las clases. Revista de didáctica ELE marco ELE, 7(8). http://marcoele.com/descar gas/7/nevado_juego.pdf

Rincic, L. y Valero M. (2002). Mínimo estimulo significativo: Velocidad y Flexibilidad. Trabajo de Grado, Universidad Abierta Interamericana, Argentina. http://imgbiblio.vaneduc.edu.ar/fulltext/files/TC043368.pdf

Rodríguez, G. (2006). La importancia del juego en educación. http://www.uneduc.cl/documentos/La\%20

Rodríguez, G. Monroy, A. (2010) El concepto de juego en relación con la actividad física. https://www.efdeportes.com/efd150/el-concepto-de-juego-en-relacion-con-la-actividadfisica.htm

Roncancio, C., y Sichacá, E. (2009). La Actividad Física Como Juego en la Educación Inicial de los Niños Preescolares. https://pdfs.semanticscholar.org/7588/a7309fac87f95dbf0d09751fd4c8d59a7 182.pdf

Serrano López, E. (2014). Efectos de los recursos materiales fungibles específicos de EF en el desarrollo de la coordinación visomotriz en centros docentes de primaria. Tesis doctoral, Universidad de Sevilla.

Tamayo, T. y Tamayo, M. (2005). El Proceso de la Investigación científica. Editorial Limusa S.A. México. 
Trujillo, F. (2010) Recursos y materiales en Educación Física. Consultado 30 enero 202. Disponible: https://www.efdeportes.com/efd140/recursos-y-materiales-en-educacionfisica.htm

Trujillo, F. (2010).Recursos y materiales en Educación Física. Recuperado de. https://www.efdeportes.com/efd140/recursos-y-materiales-en-educacion-fisica.htm

UNESCO. (1980). El niño y el juego Planteamientos teóricos y aplicaciones pedagógicas. $\begin{array}{llllll}\text { Estudios } & y & \text { documentos } & \text { de } & \text { educación, } & 34,\end{array}$ http://unesdoc.unesco.org/images/0013/001340/134047so.pdf

UNESCO. (2014) El Informe de Seguimiento de la EPT en el Mundo. http://unesdoc.unesco.org/images/0022/002261/226159s.pdf

Vargas Murillo, G. (2017). Recursos educativos didácticos en el proceso enseñanza aprendizaje. Cuadernos Hospital de Clínicas, 58(1), 68-74. http://www.scielo.org.bo/scielo.php?script=sci_arttext\&pid=S1652$\underline{67762017000100011 \& \operatorname{lng}=\text { es \& tlng=es. }}$

Vidarte, J. Sandoval, C. Alfonso M. (2011). Actividad física: Estrategia de promoción de la salud. Hacia la promoción de la salud, 16(1), 202-218. http://www.scielo.org.co/pdf/hpsal/v16n1/v16n1a14.pdf

Zabalza, M. (2006) Didáctica de la educación infantil, 4ª Edición. Madrid: Narcea.

Zampa, C. (2007). La importancia de respetar las etapas del desarrollo en deporte: una mirada desde la psicomotricidad. https://docplayer.es/12597613-La-importancia-de-respetarlas-etapas-del-desarrollo-en-deporte-una-mirada-desde-la-psicomotricidadresumen.html 